\title{
Selenium nanoparticles: An insight on its Pro-oxidant and antioxidant properties
}

\author{
Prashanth Kondaparthi, SJS Flora* and Saba Naqvi* \\ National Institute of Pharmaceutical Education and Research (NIPER- Raebareli), Lucknow, U.P., India
}

\begin{abstract}
Nanotechnology is manipulation of matter at an atomic, molecular, and supramolecular scale with particles size range in between 1 and 100 nanometres (nm) having unique properties such as high surface area, bioavailability, and reduced adverse side effects and sustained drug release. Nanomaterials of different nature such as metal (gold, platinum, palladium) organic (i.e. melanin, lignin) and metal oxides like cerium oxide nanoparticles, demonstrate intrinsic redox property which is often related with free radical scavenging property. Among various inorganic nanoparticles, the Se can be extensively exploited due to its unique antioxidant properties. The selenium is a micronutrient trace element, the antioxidant effect is chiefly due to the selenoenzymes such as thioredoxin reductase (TR) and glutathione peroxidase family (GPxs) which is having ROS scavenging activity. On the other hand, the selenium also exhibits ROS generating ability and leads to oxidative stress. The elemental selenium $\left(\mathrm{Se}^{+6}, \mathrm{Se}^{+4}, \mathrm{Se}^{-2}\right)$ having narrow therapeutic margin as compared with selenium nanoparticles which is having reduced toxicity. The selenium nanoparticles had been widely used in various oxidative and inflammatory mediatory disorders like cancer, diabetes, arthritis etc. In current review, we have discussed the antioxidant and pro-oxidant properties of selenium nanoparticles and their mechanism of action involving in reducing the generation of ROS.
\end{abstract}

\section{Introduction}

Nanotechnology is regarded as a new aspect to fight and prevent diseases by using atomic scale altering of materials. The ability to exploit the structure and function of the biosystems at the nanoscale, awaken research leading to advancement in biology, biotechnology, medicine and healthcare. The size of the nanomaterials is resemblance to that of most biological molecules and structures; consequently, these nanomaterials which can be useful for both in vivo and in vitro biomedical research and applications. Combination of the nanomaterials with biology has led to the improvement of contrast agents, diagnostic devices, physical therapy applications, analytical tools, and drug delivery vehicles [1]. Nanomaterials are auspicious nanocarriers with the extraordinary properties including high stability, soluble behaviours, thermal properties; controllable morphology and surface functionalization are substitute for traditional medicine. Among them, Selenium nanoparticle has become more diligence due to its unique antioxidant properties [2].

The Selenium is an important micronutrient trace element that plays a principle role in humans and animals and has been suspected to have essential health benefits [3]. Selenium (Se) which has been involved in the antioxidant defence systems of the liver and plays an essential role in defending against oxidative stress. Many studies explaining that Se supplementation can raises the level of enzymes such as GPx etc., prevent the augmentation of free radical species, and decrease the cellular damage. Moreover, the narrow margin between the effective and toxic doses restricted the utilization of this substance [4]. SeNPs have been used in various oxidative stress and inflammation mediated disorders like cancer, diabetes, arthritis and nephropathy with possibility therapeutic benefits due to their reduced toxicity. It has incomparable antioxidant and pro-oxidant eff ects depending on the dose, duration and the oxidation state [5]. The selenium nanoparticle which is having ROS scavenging activity is mainly credited to selenoenzymes, thioredoxin reductase (TR) and glutathione peroxidase family (GPxs) [6]. The selenium nanoparticle also having capability of producing ROS and causing toxicity [7]. The antioxidant effect of selenium mainly associated to selenoenzymes, the glutathione peroxidase family (GPXs) and thioredoxin reductase (TR). The antioxidant effect of selenium nanoparticle is well known in several studies $[3,8,9]$. The Selenium nanoparticle also has capable of producing (ROS) (prooxidant) effect [7]. In present review, we discussed about the pro-oxidant and antioxidant effect of selenium metal and its nanoparticles [10-13].

\section{Nanotechnology}

Nanotechnology mainly defined as the tailoring of materials at atomic, molecular and macromolecular scales, where properties are differed from when compared with bulk scale. Nanotechnology is a versatile field which is having notable impact in various fields such as, astronomy, chemistry, physics, medicine, nano- electronics and environmental remediation. Nanotechnology implementation in various areas of health and science, leads to benefit for human life. The nanomaterials are constituents, having size range of $10^{-9}$ in which their biological, catalytic, physical, chemical, and optical properties which are different from their bulk formulation. Scientists who are working

${ }^{\star}$ Correspondence to: Naqvi S, National Institute of Pharmaceutical Education and Research (NIPER- Raebareli), Bijnor-Sisendi Road, CRPF Base camp, P.O. Mati, Sarojini Nagar, Lucknow, U.P., 226002, India, E-mail: writetosaba@yahoo.com

Flora SJS, National Institute of Pharmaceutical Education and Research (NIPERRaebareli), Bijnor-Sisendi Road, CRPF Base camp, P.O. Mati, Sarojini Nagar, Lucknow, U.P., 226002, India, E-mail: sjsflorhotmail.com

Key words: selenium, selenium nanoparticles, pro-oxidants, antioxidants

Received: December 02, 2019; Accepted: December 16, 2019; Published: December 19, 2019 
hard to create new novel systems, nanodevices and multifunctional materials by utilizing the novel policy of nanomaterials, such as quantum size effect and their large surface to volume ratio there by reducing matter at the atomic, molecular, and supramolecular levels. The various application of the nanotechnology in various field such industry, business, medicine, solar energy, agriculture, and public health.

The nanotechnological products are presently getting much more attention and sudden changes in the fields like food, cosmetics, sporting, apparel, household products and most prominently medicines, because of their cost-effectiveness, novelty, and high sustainability.

Nanotechnology has expanding its applications in cancer treatment, healthcare, medical defence, electronics, consumer products, spaceflight, environment, chemical sensors.

\section{Nanoparticles}

The nanomaterials which is having particle size less than $100 \mathrm{~nm}$ is generally regarded as nanoparticle which is generally engineered nanoparticle. It brought a great revolution in the industrial sector. Because of their different physicochemical and electrical properties, the nanoparticles have more attention in the fields of electronics, biotechnology, aerospace engineering and medicinal field. Because of their important contribution to the medicinal field NPs are mainly used as a new delivery system for drugs, DNA, proteins, and monoclonal antibodies. The nanoparticles were prepared from their bulk counterpart materials such as metal and non-metal, mono polymers etc., whereas polyethylene glycol, liposomes, and dendrimers are widely used in theranostics [10]. Figure 1 showed different types of nanoparticles (NPs) which are commonly used nanoparticles in various biomedical applications.

The various type of nanoparticle which can be used as a therapeutic agents and drug delivery carriers such as polymers, liposomes, dendrimers, metal nanoparticles ( $\mathrm{Fe}, \mathrm{Se}, \mathrm{Ag}, \mathrm{Au}, \mathrm{Ce}, \mathrm{Cu}, \mathrm{Eu}$,) etc. The main properties of the nanoparticles such as high surface area, small size, solubility surface chemistry, surface charge and multifunctionality makes them unique. By delivering of the therapeutic molecules, the NPs have been proved as a drug carrier. Due to their application in various fields these nanoparticles have been used in clinical practice and medical research employed for the diagnosis, treatment, monitoring and control of biological systems as it is called nanomedicine. The NPs can solve many of the pharmacokinetic (PK) problems that may associate with many drugs that has been used in a variety of disease classes. NPs improves the therapeutic efficiency of ionised drugs; enhance the penetration of water-soluble compounds, proteins, peptides, siRNA, miRNA, DNA, vaccines, and many other biological therapeutics substance. Via alteration of their surface using suitable targeting ligands or moieties, nanoparticles unable the drug delivery system much effective and target specific. Metallic nanoparticles such as $\mathrm{Se}, \mathrm{Si}, \mathrm{Ti}, \mathrm{Ag}, \mathrm{Au}, \mathrm{Ce}, \mathrm{Fe}$ and $\mathrm{Zn}$ has an important role in the field of nanotechnology because of their distinctive opportunity not only as theragnostic agents but also excellent potential as carriers for chemotherapeutic agents, siRNA, proteins etc [5].

\section{Nanotoxicity}

Humans are exposed to a variety of particles which are small in size and again this new emerging field of nanotechnology has both pros and cons on human health. Because of their small size, these NPs can easily enter the human body and penetrates various biological barriers and penetrates to various sensitive organs. The scientists have been proposed that NPs having less than $10 \mathrm{~nm}$ size act exactly as a gas that can enter human tissues easily and may disrupt the normal cell physiological environment. Many preclinical and clinical studies have shown that after inhalation and through oral exposure, these NPs in addition to lungs and gastrointestinal tract it is distributed to the liver, spleen, heart, and brain. For clearing these nanoparticles out of the body, the components of the immune system are activated. Because of the larger half-life of the nanoparticle which is estimated to be 700 days in human lungs that posing a consistent threat to respiratory system. During metabolism, the nanoparticles are deposited in the liver tissues. The toxicities of the nanoparticles are inversely proportional to their size because the smaller size can cause more toxic to human health when compared with larger size particles. Because of their characteristic physicochemical properties in various biological systems, unexpected health outcomes of NPs were illustrious to scientists. So, to bridge the gap of knowledge and to counter the toxicity issues related to NPs, various approaches aiming to safe use of NPs is the need of an hour [10] (Figure 2).

\section{Selenium}

Selenium (Se) is an essential dietary trace element which presents in human being (Fang, et al. 2018). In humans, Se has the narrowest margin levels ranges between dietary deficiency $(<40 \mathrm{mg} /$ day $)$ and toxic levels (>400mg/day). The dietary selenium intake reference has set

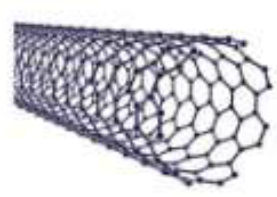

Nanotubes

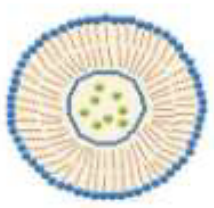

Liposomes
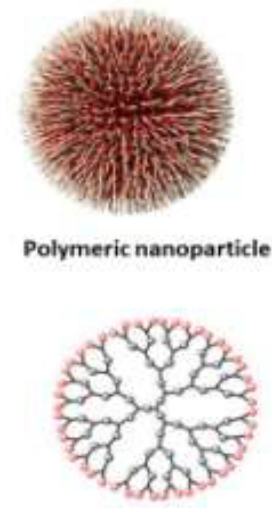

Dendrimer
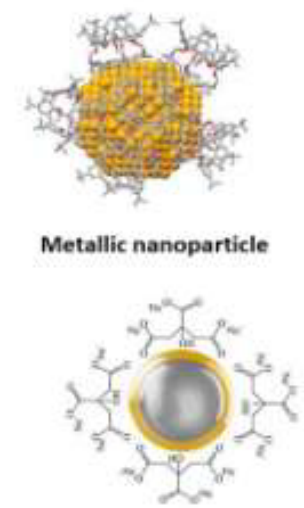

Magnetic nanoparticle

Figure 1. Different types of nanoparticles (NPs) which are commonly used nanoparticles in various biomedical applications 


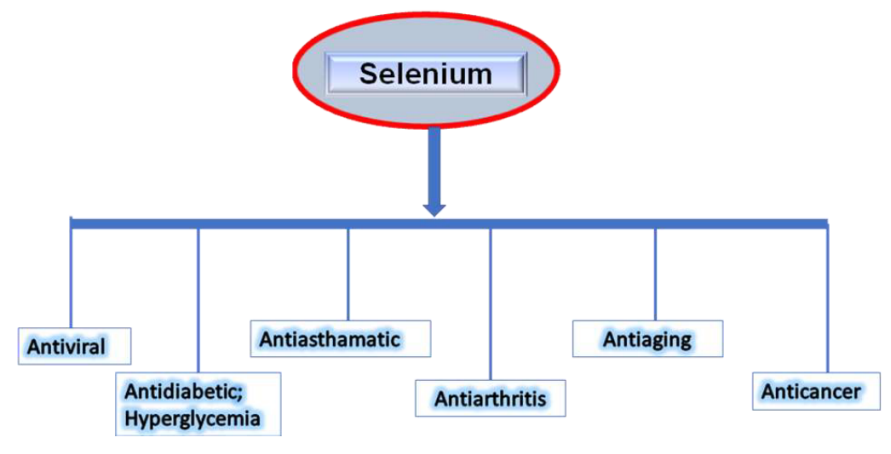

Figure 2. Health benefits of selenium

between the range of $30-55 \mathrm{mg} /$ day by international agencies [3]. Se which is a building block of the selenocysteine, that mainly involved in the synthesis and catalytic function of selenoproteins such as peroxidases and reductases [11]. The selenium believed to incorporated into selenoproteins as selenocysteine $(\mathrm{SeC})$ which is vital part of active center of their enzymatic activities. Many selenoproteins believed to be having oxidoreductase activity which play important role in regulation of the physiological redox balance. Due to the narrow therapeutic window and toxicity margins the selenium is widely replaced by the selenium nanoparticle which is believed to be having wide therapeutic window and reduced toxicity which also having the optimized body distribution [5].

Selenium is a metalloid having different oxidation states, viz. selenite $\left(\mathrm{SeO}_{3}\right)^{2-}$, selenite $\left(\mathrm{SeO}_{4}\right)^{2-}$, selenide $(\mathrm{Se})^{2-}$ and elemental selenium $(\mathrm{Se} 0)$. Inorganic selenium mainly occurs in the soil, while organic selenium mostly occurs in soil, air and plants, which impulse that the most of the selenium normally from the environment. The plants can absorb the selenium in the form of sodium selenite from the soil, which mostly converted into selenomethionine, with some trace amounts of SeCys, methyl-SeCys and $\gamma$-glutamyl-Se-methylcys can also formed. The selenium is preferentially present as selenoproteins (glutathione peroxidase), which can also complex to amino acids (selenocysteine and selenomethionine). Presently there are 25 selenium proteins which are known to play a role as antioxidants, with selenite, selenomethionine, methylselenocysteine and selenocysteine are the compounds which are most studied ones, because of their application in disease prevention and therapy. Many Studies revealed that the elemental selenium is less toxic and preferential biological activity compared with its other forms $[1,12]$. The elemental selenium mainly involved in the antioxidant defines system that play a vital role in protecting against oxidative stress. In several studies they showed that the Selenium can upregulates the level of enzymes such as GPx etc., with the supply of Selenium and reducing the cellular damage by preventing the accumulation of free radical species. However, due to its narrow margin between the toxic and effective doses which restricted the use of this selenium. The Se0 which believed to be having more attention due to its low toxicity and more bioavailability when compared with

Se (IV) and Se (VI), meanwhile they both having free radicals capturing ability. Due to the poor water solubility and their ability to convert into a grey analogue which is not only thermodynamically stable, but also biologically inert It is difficult to be apply in food and medicine fields for selenium because of its poor water solubility and ability to convert into a grey analogue. The water solubility of the selenium nanoparticle can be enhanced by reducing their size and increasing their surface area with application of the nanotechnology [4].
When selenium is supplied to the elderly volunteers in Arizona that can raises the $\mathrm{T}$ cell count. There was increased in $\mathrm{CD}^{4+} \mathrm{T}$ cell count was observed accompanied by the increased cytotoxicity of natural killer cells (NKCs). The selenium can improve the cell mediated immune responses when it is supplied in the form of sodium selenite to head and neck cancer patients during radiation and surgery. The selenoproteins are required for the normal function of the activated $\mathrm{T}$ cells and the $\mathrm{T}$ cells are sensitive to ROS when the deficiency of selenoproteins occurs, it leads to ROS elevation, so selenoproteins, cannot proliferate in response to the $\mathrm{T}$ cell receptor stimulation. Selenium depletion leads to irreversible brain injury. The Selenium can deliver to brain by specific selenoprotein called selenoprotein $\mathrm{P}$ by binding to apoER2 receptor (a member of the lipoprotein-receptor family). The selenoprotein $\mathrm{P}$ also believed to be improving the neuronal survival and also prevent cell death caused by beta-amyloid accumulation $[13,14]$.

\section{Selenium nanoparticles}

The selenium having a narrow therapeutic window and the toxicity margins while, the selenium nanoparticle (SeNPs) having unusually reduced toxicity. The various uses of SeNPs have been employed in various oxidative stress and inflammation mediated disorders like cancer, arthritis, diabetes and nephropathy with a possible therapeutic benefits. The SeNPs may act as a carrier for carrying a drug to the site of action. The mortality caused by acute toxicity associated with the Se, can be minimized by the use of SeNPs up to four times in a rodent model. The high dosage of Se can cause liver injury which can be believed to be reduced by using SeNPs as evident of hepatotoxicity biomarkers. SeNPs can exhibit charismatic anticancer activity and reduced toxicity when we linked to diff erent Se species (selenite, selenite and organic selenium). The SeNPs gain much attention in several pathological conditions like, inflammatory disorders, cancer, diabetes and drug induced toxicities. When nano selenium compared to inorganic and organic Se compounds, it having more bioavailability and biological activity. Figure 3 demonstrates the therapeutic benefits of SeNPs including their anticancer, antioxidant, anti-inflammatory and anti-diabetic action.

\section{Pro-oxidant effect of selenium nanoparticles}

Among all the inorganic nanoparticles which has been explored to induce cytotoxicity in cancer cells one of the most exploited nanoparticle is SeNPs. The SeNPs approaches believe to be help in fighting with the drug resistance problem and minimizing the toxicities that occurs with the chemotherapeutic agents. The SeNPs believed to be as a carrier for chemotherapeutics drug and delivered to the target site. It is believed that the NPs were significantly localized inside the malignant cells and caused the formation of the reactive oxygen species (ROS) therefore, causing cytotoxicity. The SeNPs may enter through receptor mediated endocytosis. Because of the acidic $\mathrm{pH}$ state with redox imbalance these malignant cells will enables the selenium nanoparticles to exhibits pro-oxidant effect by generating free radicals on one side, they can cause mitochondrial membrane disruption which can cause leakage of mitochondrial $(\mathrm{Mt})$ proteins and also causes endoplasmic reticulum (ER) stress.

Because of the mitochondrial damage there is a leakage of proteins which can trigger apoptosis (activation of caspases). This make the cellular stress due to the activation of multiple molecular pathways that including NFkB, MAPK/Erk, Wnt/ $\beta$-catenin, PI3K/Akt/mTOR and apoptotic pathways. The NFkB pathway can interrupt cellular homeostasis due to the stimulation of the inflammatory and oxidative stress signalling. These pathways are important in oncogenic signalling 
and their regulations by SeNPs which can causes reduced cellular proliferation and obstruct the growth promoting signalling in the proximity of tumour surrounding microenvironment. In addition, the SeNPs can also having capable of reducing the angiogenic signalling in tumour cells which can considerably minimise growth and proliferation. Combination of these troublesome cellular events which makes the DNA damage and causing cell cycle arrest that ultimately results in cellular death. Meanwhile, the genetic analysis of various diseased and treated animals showed that nanoparticles augmenting the expression of ING3 (which interacts with p53 and induces apoptosis), aldo-ketoreductase1B10 (Akr1b10) and decreased the levels of Foxp1 gene (Forkhead box, class $\mathrm{O}$ which normally believed to be involved in proliferation of hepatic stellate cells). The SeNPs can reduces the expression of the CD44 and necrosis in MCF-7 cells by apoptosis induction which caused by the reduction of the adhesion force caused disorganization and dysregulation of the intracellular cytoskeleton F-actin in MCF-7 cells. Study reported that SeNPs believed to be inhibiting the matrix metalloprotein-2 expression which involved in tumour invasion, metastasis and angiogenesis in fibro-sarcoma cell lines (HT1080) [5]. Figure 4 demonstrates the Pro-oxidant mechanism of selenium nanoparticles which exhibits that nano selenium is reduced by the Trx/TrxR/Trx/GSH/GR/GRx pathway and leads to formation of selenide ( $\left.\mathrm{Se}^{-}\right)$anion by the utilization of the $\mathrm{NADPH}+\mathrm{H}^{+}$and further formation of free radical $\left(\mathrm{O}_{2}^{-)}\right.$occurred which leads to reactive oxygen species (ROS).

\section{Antioxidant effect of selenium nanoparticles}

The antioxidant effect of selenium nanoparticle is mainly associated to selenoenzymes, the glutathione peroxidase family (GPXs) and thioredoxin reductase (TR). The GPXs has a capacity to detoxify extended range of peroxides, such as $\mathrm{H}_{2} \mathrm{O}_{2}$, phospholipid hydroperoxide, fatty acids hydroperoxides, and hydroperoxyl groups of thymine. The TR (thioredoxin reductase), in combination with its substrate thioredoxin, which forms a redox system, that having multiple functions, including a detoxification reaction [6]. The ROS and RNS are the most common free radicals that present in nature. These free radicals are derived from oxygen such as peroxyl radical, superoxide radical, per hydroxyl radical, hydroxyl radical and also non-free radical species such as hydrogen peroxide and singlet oxygen which can also having capable of generating free radicals, while that of reactive nitrogen species such as peroxynitrite, nitric oxide nitrogen dioxide. The free radical species are unstable due to the presence of one or unpaired electron in their outermost shell and remove electrons

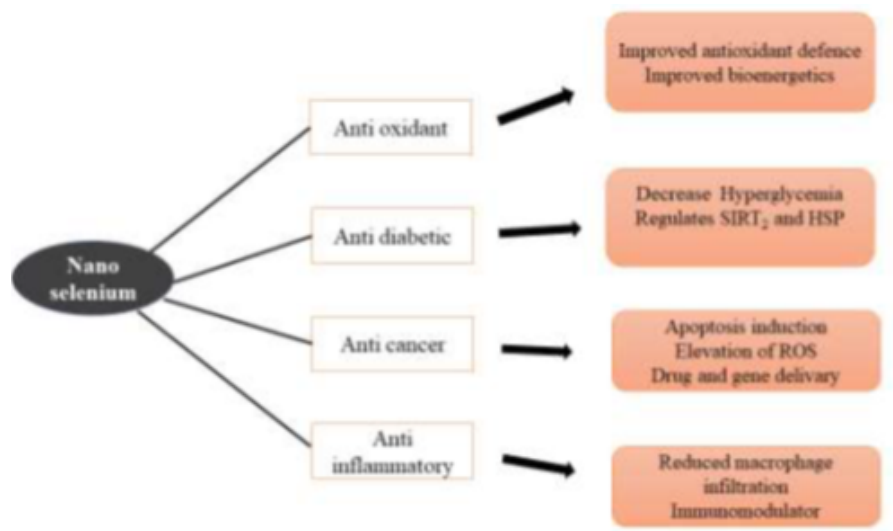

Figure 3. The various therapeutic benefits of selenium nanoparticles including anticancer, antioxidant, anti-inflammatory and anti-diabetic action

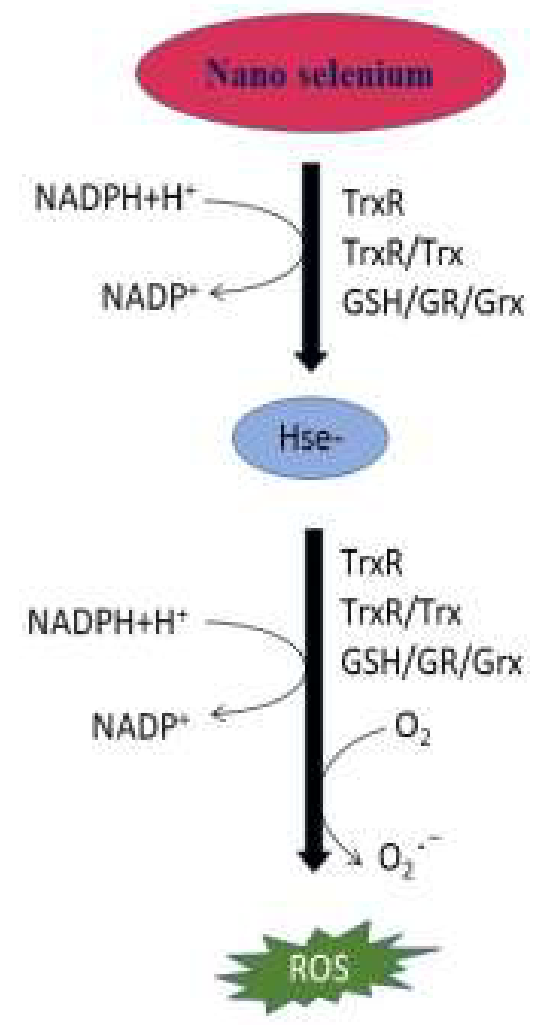

Figure 4. Pro-oxidant mechanism of selenium nanoparticle which include that nano selenium is reduced by the $\operatorname{Tr} / \operatorname{TrxR} / \operatorname{Trx} / \mathrm{GSH} / \mathrm{GR} / \mathrm{GRx}$ pathway which leads to formation of selenide $\left(\mathrm{se}^{-}\right)$anion by the utilization of the $\mathrm{NADPH}+\mathrm{H}^{+}$and further formation of free radical $\left(\mathrm{O}_{2}\right)$ occurred, which lead to the formation of ROS

from other compounds to attain stability which leads to chain reaction cascade generating more reactive species. Several studies revealed that the physiological system having high level of ROS which exert oxidative stress that may cause many diseases by disrupting or damaging lipids, proteins and DNA. To protect against this oxidative stress, the biological system has a set of antioxidant enzymes such as glutathione peroxidase (GPx), thioredoxin reductase (TrxR) and iodothyronine deiodinases (IDD which play important role in protecting from oxidative stress and the selenium is important as it is the main component of these antioxidant enzymes. When compared with Se-methyl, selenocysteine and selenomethionine and selenite, the selenium nanoparticles can increase the activity of selenoenzymes with equal efficacy and less toxicity [5]. NanoSe which can be used as effectively in enhancing the level of glutathione peroxidase and thioredoxin reductase that is comparable with selenomethionine with reduced toxicity. The SeNPs believed to be reduced the $\mathrm{K} 2 \mathrm{Cr} 2 \mathrm{O} 7$ induced oxidative stress in some thyroid gland and restored the T3, T4, catalase, superoxide dismutase (SOD), and GSH levels in treated animals. Moreover, the SeNPs protects the cellular structure, organisation and which also prevented the cell damage and also it inhibits the changes that observed in the thyroid's gland [5] (Figure 5).

\section{Conclusions}

The selenium is an essential micronutrient trace element which is having a unique antioxidant effect via selenoenzymes such as Glutathione peroxidase $(\mathrm{Gpx})$, Thioredoxin reductase (TrxR) and Iodothyronine deiodinases (IDD). These enzymes protect our body from oxidative stress. The selenium nanoparticles is reported as less toxic as compared to selenium which having narrow margin of toxicity. 


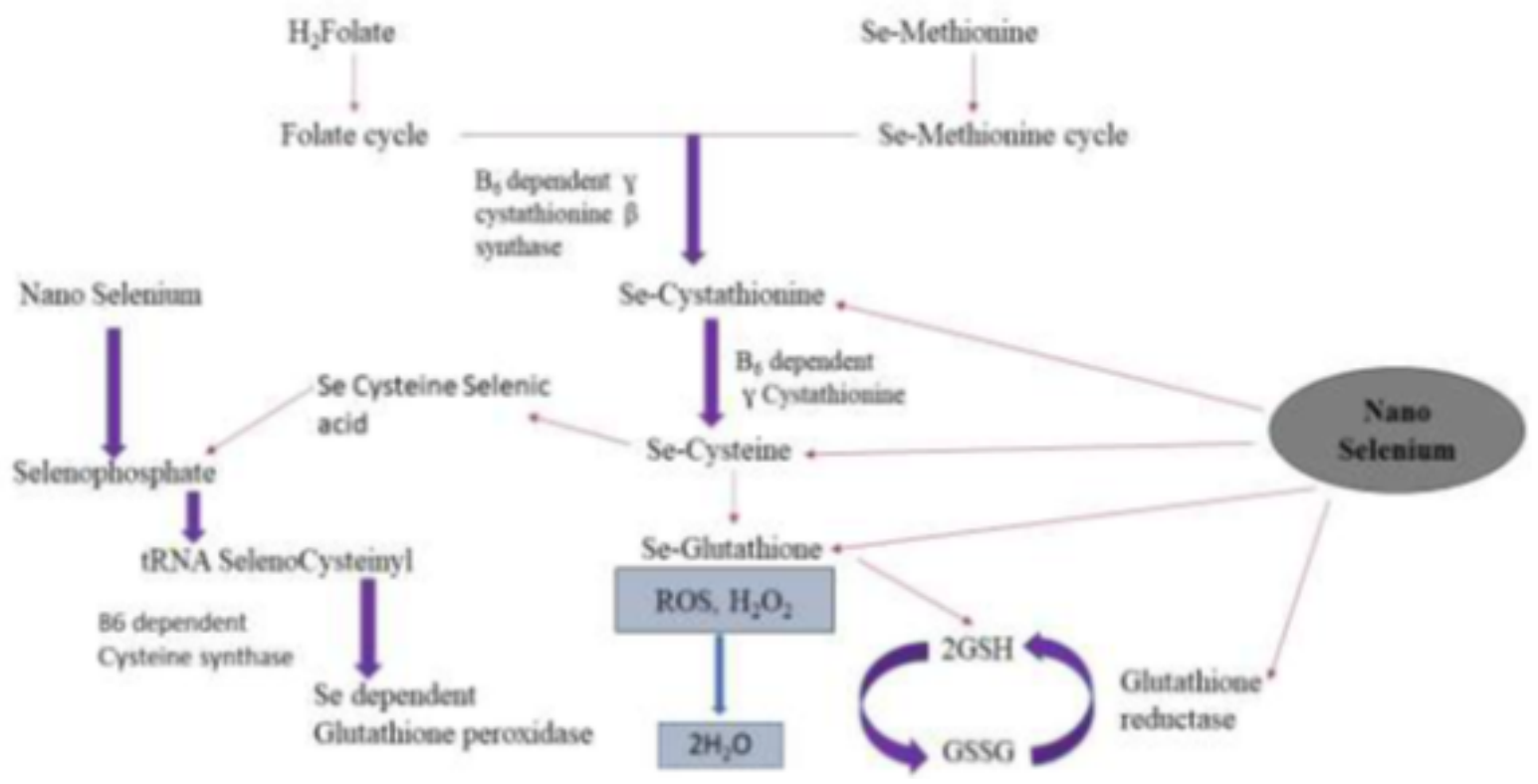

Figure 5. Antioxidant mechanism of selenium nanoparticle

The selenium nanoparticles are having unique antioxidant and prooxidant effects, depending on their dose, duration, frequency and oxidation state. On the hand, selenium and its nano form also having the capability to produce ROS which presently used as an anticancer property and showing synergistic effect. The safety profile of the selenium nanoparticle is not yet clearly defined which limits their application. Hence in near future, we have to generate more scientific data on their nanotoxicity as well as their therapeutic values.

\section{Acknowledgements}

SJS Flora and SN are grateful to Department of Pharmaceuticals, Ministry of Chemical and Fertilizes, Govt. of India, S.N. was further supported by Women Scientist Scheme (WOS-A), Department of Science and Technology, Government of India (grant no. SR/WOS-A/ LS-1224/2015). PK was supported by GPAT, AICTE scholarship.

\section{Conflict of interest}

The authors declare no competing conflict of interest.

\section{References}

1. Singh M, Singh S, Prasad S, Gambhir IS (2008) Nanotechnology in medicine and antibacterial effect of silver nanoparticles. Digest Journal of Nanomaterials and Biostructures 3: 115-122.

2. Li Y, Guo M, Lin Z, Zhao M, Xia Y, et al. (2018) Multifunctional selenium nanoparticles with Galangin-induced HepG2 cell apoptosis through p38 and AKT signalling pathway. Royal Society open science 5: 180-509.

3. Saif-Elnasr M, Abdel-Aziz N, El-Batal AI (2019) Ameliorative effect of selenium nanoparticles and fish oil on cisplatin and gamma irradiation-induced nephrotoxicity in male albino rats. Drug Chem Toxicol 42: 94-103.
4. Zhai X, Zhang, C, Zhao G, Stoll S, Ren F, et al. (2017) Antioxidant capacities of the selenium nanoparticles stabilized by chitosan. Journal of Nanobiotechnology 15: 4.

5. Khurana A, Tekula S, Saifi MA, Venkatesh P, Godugu C (2019) Therapeutic applications of selenium nanoparticles. Biomed Pharmacother 111: 802-812.

6. Zhang JS, Gao XY, Zhang LD, Bao YP (2001) Biological effects of a nano red elemental selenium. Biofactors 15: 27-38.

7. Zhao G, Wu X, Chen P, Zhang L, Yang CS, et al. (2018) Selenium nanoparticles are more efficient than sodium selenite in producing reactive oxygen species and hyperaccumulation of selenium nanoparticles in cancer cells generates potent therapeutic effects. Free Radic Biol Med 126: 55-66.

8. Bhattacharjee A, Basu A, Ghosh P, Biswas J, Bhattacharya S (2014) Protective effect of Selenium nanoparticle against cyclophosphamide induced hepatotoxicity and genotoxicity in Swiss albino mice. J Biomater Appl 29: 303-317.

9. Sadek KMV, Lebda MA, Abouzed TK, Nasr SM, Shoukry M (2017) Neuroand nephrotoxicity of subchronic cadmium chloride exposure and the potential chemoprotective effects of selenium nanoparticles. Metab Brain Dis 32: 1659-1673.

10. Bahadar H, Maqbool F, Niaz K, Abdollahi M (2016) Toxicity of nanoparticles and an overview of current experimental models. Iran Biomed J 20: 1.

11. Hadrup N, Loeschner K, Skov K, Ravn-Haren G, Larsen EH, et al. (2016) Effects of 14-day oral low dose selenium nanoparticles and selenite in rat-as determined by metabolite pattern determination. Peer J 4: 2601.

12. Maiyo F, Singh M (2017) Selenium nanoparticles: potential in cancer gene and drug delivery. Nanomedicine 12: 1075-1089.

13. Majeed W, Zafar M, Bhatti A, John P (2018) Therapeutic potential of selenium nanoparticles. J Nanomed Nanotechnol.

14. Fang X, Li CE, Zheng L, Yang F and Chen T (2018) Dual Targeted Selenium Nanoparticles for Synergistic Photothermal Therapy and Chemotherapy of Tumors. Chemistry-An Asian Journal 13: 996-1004.

Copyright: $@ 2019$ Kondaparthi P. This is an open-access article distributed under the terms of the Creative Commons Attribution License, which permits unrestricted use, distribution, and reproduction in any medium, provided the original author and source are credited. 